\title{
Identification and Analyses in Optical Light of Gamma-Ray Sources with Astronomical Archival Plates
}

\author{
René Hudec ${ }^{1,2}$ and Miloš Klíma ${ }^{2}$ \\ ${ }^{1}$ Astronomical Institute, Academy of Science of the Czech Republic, 25165 Ondřejov, Czech Republic \\ ${ }^{2}$ Faculty of Electrical Engineering, Czech Technical University in Prague, 16636 Prague, Czech Republic
}

Correspondence should be addressed to René Hudec, rene.hudec@gmail.com

Received 13 June 2009; Accepted 2 September 2009

Academic Editor: Alberto J. Castro-Tirado

Copyright (C) 2010 R. Hudec and M. Klíma. This is an open access article distributed under the Creative Commons Attribution License, which permits unrestricted use, distribution, and reproduction in any medium, provided the original work is properly cited.

\begin{abstract}
The ESA INTEGRAL satellite (International Gamma Ray Laboratory) launched in October 2002 continues to deliver valuable data about the gamma-ray sky. Nearly 450 gamma-ray sources have been detected so far mainly by the IBIS onboard instrument, and others are expected in the future. The first 3.5 years of INTEGRAL public and Core Program data have revealed more than 400 sources and this number is expected to increase to more than 500 in the future (Bird et al. 2007). Alternative method to identify and to analyze INTEGRAL gamma-ray sources using optical light and astronomical archival plates is described, together with additional results from analyses of high-energy sources in these databases.
\end{abstract}

\section{Identification of High-Energy Sources}

Only a fraction of the newly detected INTEGRAL hard Xray/gamma-ray sources are either known sources or have been identified and classified already. From the 56 newly by- INTEGRAL detected sources (IGR sources), only $20 \%$ have already firm classification, mostly with Cataclysmic Variables (CVs), AGN, High Mass X-ray Binaries, Low Mass $\mathrm{X}$-ray Binaries, Black Hole Candidates, and Anomalous Xray Pulsars [1]. One of the methods applied in the past is the identifications by spectroscopy, which proved recently some new and interesting identification of INTEGRAL gamma-ray sources such as newly detected symbiotic and cataclysmic variables (e.g., [2]).

\section{Limitations of Spectroscopic Method}

Although successful, this method based on optical and/or NIR spectroscopy has some limitations. First, it can be hardly applied for particular types of transients and even recurrent transients. Secondly, it requires access to dedicated large aperture telescopes and spectrographs and this is mostly limited, and expensive. Moreover, it can be laborious in the cases of large error box and crowded field, and this is quite a common situation as most of the INTEGRAL fields indeed lie in the Galactic Plane region. And in some specific cases, only the spectral information alone is sometimes not enough for reliable classification of the objects.

\section{The Photometric Alternative}

In this paper, we discuss an alternative method to identify the still nonclassified INTEGRAL gamma-ray sources and newly detected INTEGRAL sources in the future (and of other high-energy satellites in general). This method is based on the fact that (1) many of gamma-ray sources identified and classified so far do have optical counterparts, in many cases brighter than magnitude 18, and (2) a significant fraction of these sources is variable both in gamma-rays as well as in optical wavelengths. Both CCD images covering the error boxes of the sources taken preferably by robotic telescopes over at least few months time interval and archival sky patrol plates can be used in this case. 


\section{The Motivation}

The ESA INTEGRAL satellite focuses on the Galactic Centre region as well as on Galactic Plane Scans (GPS). Numerous new gamma-ray sources have been found in these regions by INTEGRAL, and only a fraction of these sources was successfully identified. The identification of INTEGRAL sources indicates that most of them have variable optical counterparts brighter than magnitude 18. The recent progress in classification of newly detected INTEGRAL sources has indicated that the fraction of Cataclysmic Variables and Symbiotic Stars is larger than originally expected (32 at the time of writing this paper). These sources are usually optically bright with magnitudes $10-18$ and can be easily and effectively studied at optical wavelengths by groundbased telescopes or, alternatively, by archival databases as described in this contribution. In addition to identification and classification, another goal is to provide light curves for more detailed scientific analyses. This is also an important task as the newly identified IGR sources only rarely have an optical light curve available.

\section{Suitable Databases}

The Sonneberg Field Patrol and Leiden/Johannesburg Franklin Adams Plates represent a suitable database for identification and analysis of INTEGRAL sources (see Figure 1). They both provide numerous data for regions along the Galactic Plane. In addition, the southern sky patrol plates located at the Bamberg Observatory, Germany, represent a suitable additional source of data.

\section{Sonneberg Field Patrol}

The Sonneberg Observatory Field Patrol covers selected northern regions along the Galactic Plane (but, also, other fields) by numerous (typically 50-500) astrograph plates. Typical Field of View (FOV) is $10 \times 10 \mathrm{deg}$ and the typical limiting magnitude is $B \sim 17$. In exceptional cases, also lowdispersion spectral plates are available. This kind of data is valuable for analyses related to the ESA Gaia project, as it will provide BP and RP photometric data in the form of ultralow dispersion spectra. The Sonneberg plates can be scanned as the observatory posses plate scanners, and, moreover, the plate catalogue is available as a file, hence searches in the plate catalogue are easily based on developed software package.

\section{Leiden/Johannesburg Franklin Adams Plates}

These plates were taken in Johannesburg by the high quality Franklin Adams refractor (Taylor, 1904) in years 1923-1952 within the project originated by Professor E. Hertzsprung and are located in Leiden. The plates cover selected fields along the southern Galactic Plane as well as the Galactic Centre. The typical number of plates per field is 300-400; FOV is $10 \times 10 \mathrm{deg}$ and limiting magnitude 17 .

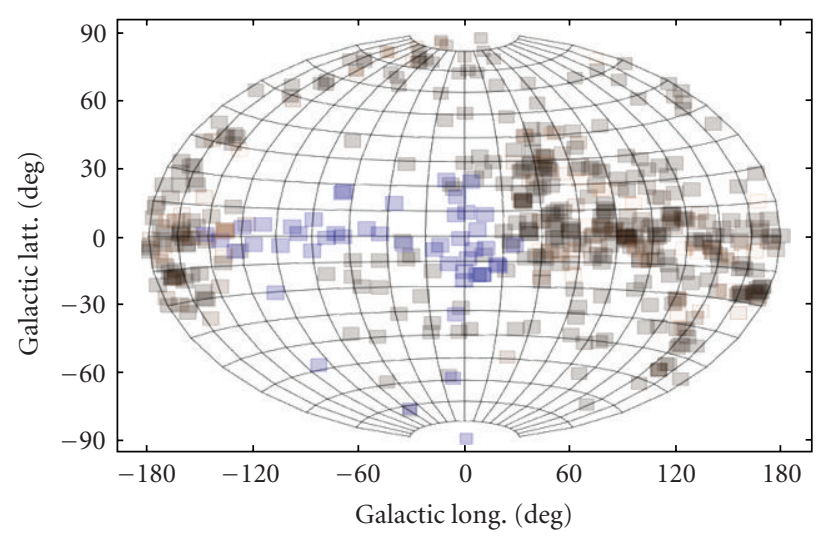

Figure 1: Distribution of Sonneberg Field Patrol Fields and Franklin Adams Fields (blue) in Galactic coordinates. The densely covered fields are darker.

Unfortunately, the plate catalogue is available only as a hard copy at the Leiden Observatory, and, moreover, there is no dedicated plate scanner available.

\section{Bamberg Observatory Plates}

The third most suitable plate archive is located at the Bamberg Observatory in Germany, and represents a systematic southern sky patrol up to lifting magnitude 15 to 15.5 . Hence all objects are covered within this limiting magnitude, and the typical number of plates available for particular position is about 200. The preference is plate catalogue as searchable file as well as available plate scanner.

\section{The Plate Analysis}

Using the data material mentioned here, the optically identified INTEGRAL sources with objects brighter than mag 17 can be investigated for long-term changes covering 10-50 years. In addition, these data can be used to search for new optical identifications of nonclassified INTEGRAL sources on hand of their optical variability. The same is valid also for optical counterparts of other high-energy (HE) sources.

For plate analyses, various methods can be used. As most of the plate archives were not yet fully scanned, the most effective method at moment is combination of on site analyses, for example, by an optical plate microscope (transmitted light), with computer analyses of digitized plates, depending on the nature of the task to be performed.

A few additional prospects are listed as follows: analyzing the light curves for flares and flaring activity, trying to fit the flare profiles, trying to look for possible periodicities and recurrences, studying of colours and colour changes with time, with consequent physical discussions and interpretations. Correlations with other objects, with related conclusions toward physical processes and physical models. 


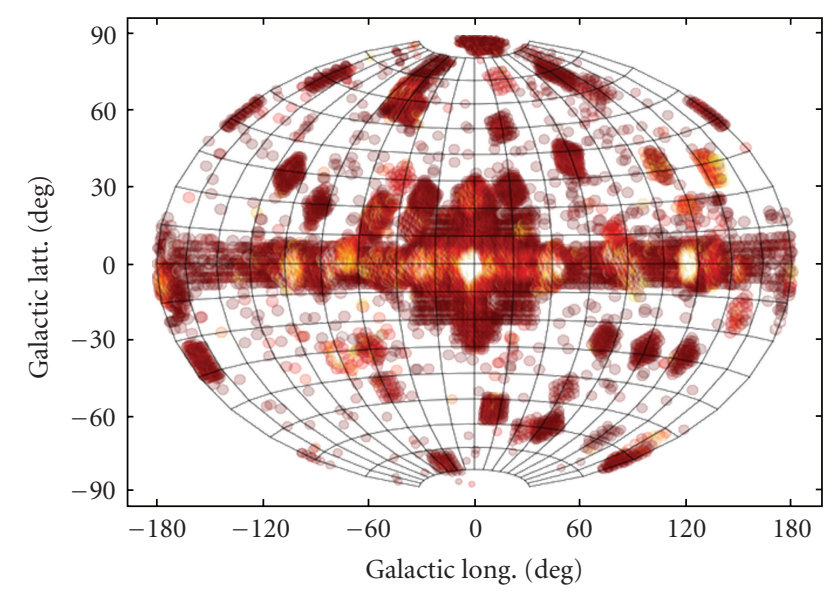

FIGURE 2: The coverage of INTEGRAL IBIS for revolutions 1-430.
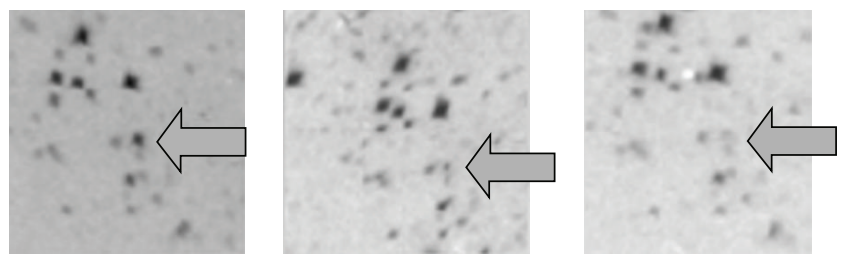

FIGURE 3: IGR J12349-6434 = RT Cru, hard X-ray symbiotic star, on Leiden Franklin Adams Plates. INTEGRAL gamma-ray source visible on astronomical plates taken by 100 years old optical telescope. Violent (amplitude 3 magnitudes) optical brightness variations about 80 years ago.

\section{Galactic Centre}

A particular interest is to be devoted to regions close to Galactic Centre where the INTEGRAL satellite coverage is large and where numerous HE sources are located, and still new sources are discovered (see Figures 2-5). This area is also heavily covered by archival plates at particular observatories (e.g., Leiden, Franklin Adams plates taken in Johannesburg). On the other hand, the crowded nature of fields near the Galactic Centre represents an additional problem especially in case of deep plates/images.

\section{GRBs Analyses}

The archival astronomical plates can be used also for study of optical afterglows (OAs) and optical transients (OTs) related to gamma-ray bursts (GRB). However, it should be taken into account that not only X-ray and gamma, but also the optical sky is highly variable even on short-term timescales. Not only do optical transients and optical afterglows of GRBs contribute but also a rich variety of transient astrophysical phenomena resulting in fast optical transients-such as flare stars, flares on AGN, novae and other types of variable stars, $\mathrm{SNe}$, and so forth-appeal on astronomical emulsion. Some of them are rare events and their detection requires wide fields ( $20 \mathrm{deg}^{2}$ or more) and prolonged ( $>1$ year of total cummulative time) monitoring intervals.
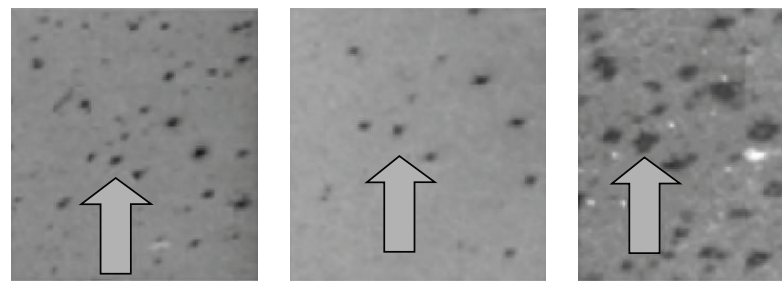

FIGURE 4: IGR J10109-5746, hard X-ray symbiotic star. Optical variability on Franklin Adams plates, field 10, Leiden, year 1928.
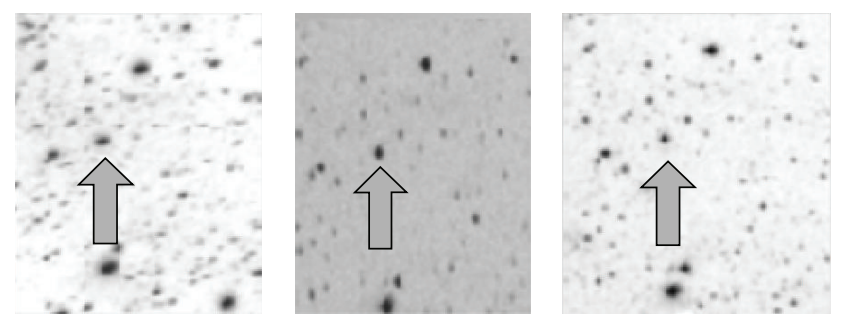

FIGURE 5: IGR J11215-5952 High-Mass X-ray Binary detected in gamma-rays by INTEGRAL and visible in optical light on historical F. A. plates (various epochs).

11.1. OTs on Astronomical Archival Plates. What is the origin of OTs found within the astronomical plate analyses of GRB error boxes? At least some of them were confirmed as real astrophysical triggers. On the other hand, relation to GRB was not confirmed. However, some of them represent very probably rare large, amplitude flares of faint AGNs.

There is unprecedented large time coverage of used plates: up to 3.2 years of continuous monitoring of a particular position collected and analysed-more than any other astrophysical search before. A question arises, namely: could indeed no relation to GRBs be established? In recent understanding, the GRB origin is in star forming regions (SFR) in distant galaxies. GRBs are not confirmed as recurrent events. However, the historical OTs found at positions of GRBs may represent another triggers from the same SFR or the same galaxy, separated in time by more than 50 years. Below we discuss some objects found on astronomical emulsions during archival GRB searches.

11.2. OT Triangulum (Figure 6). The OT Triangulum represents an example of OT of unknown origin found by a chance on astronomical archival plates: $B=10.5 \mathrm{mag}$, more than 6 mag above the plate limit, according to Hudec et al. [3]. Despite of the possible presence of plate faults, there are developed methods (such as reflected light or confocal microscopy) able to reliably exclude these false triggers. One has to be careful in elimination of background triggers such as aircraft and satellite glints, and so forth. However, the OT shown here exhibits all attributes of a real OT of an astrophysical origin.

11.3. OT GRB781119. This object was discovered by B. Schaefer inside the error box of GRB781119 as the first possible OT related to a gamma-ray burster (Schaefer, 1981 


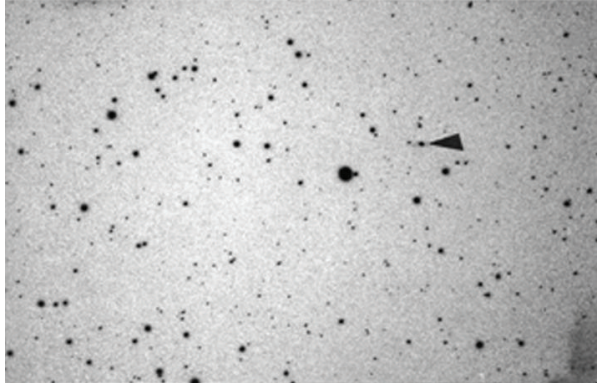

(a)

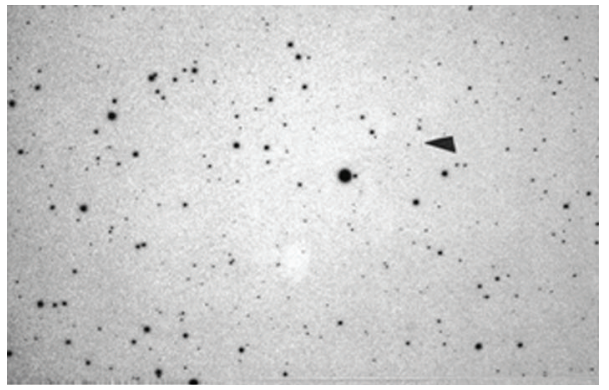

(b)

Figure 6: The OT Triangulum. Above the discovery plate with the OT (estimated magnitude $B \sim 10.5$ ) indicated, below a comparison plate with no object at the position. Astrograph plate from the Sonneberg Observatory archive, limiting magnitude 17.

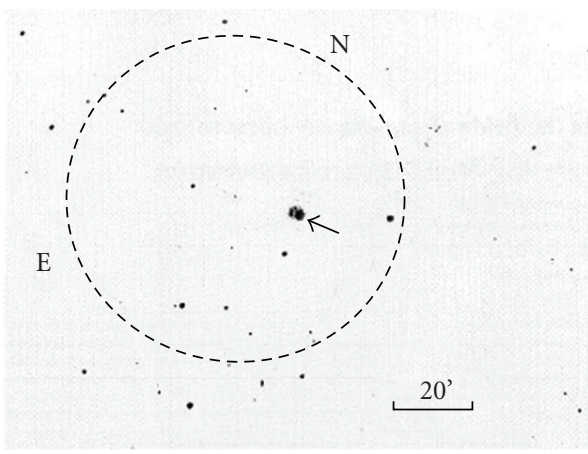

FIgURE 7: The OT inside the error box of GRB910219.

[4]). Later, there were doubts by Zytkov pointing out the alternative explanation as a plate fault. To explain this controversy, the original plate was investigated in detail by reflected light microscopy and was confirmed as real object by Hudec et al. $[5,6]$. The object relation to GRB is not clear. In fact, the OT could be caused by a blue large amplitude flare from a nearby 18 magnitude quasar.

11.4. OT GRB790325b. This object represents multiple OT from the position close to GRB790325b. It was found on astronomical archival plates (5000 plates used with total cummulative exposure time $7000 \mathrm{hrs}$ ). The OT was confirmed as real object by method of grain depth distribution [7]. A faint blue object was found on the OT position,

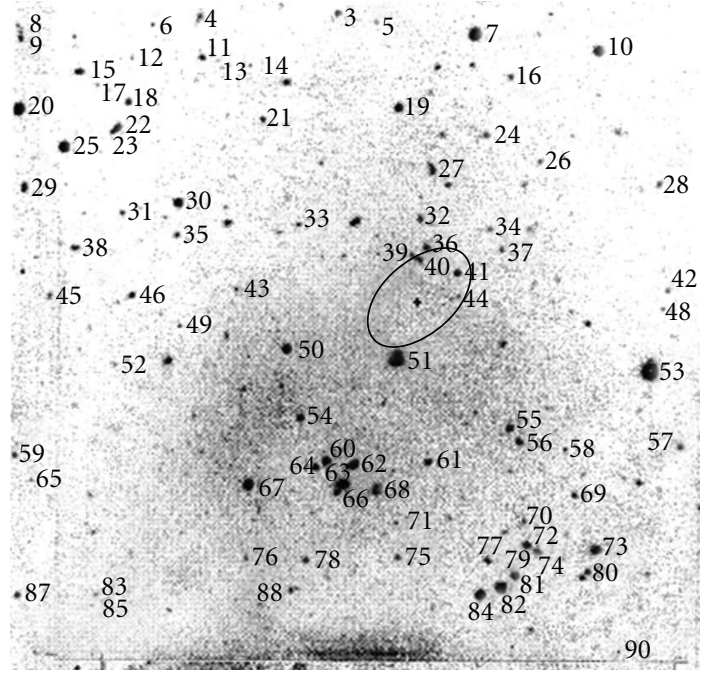

FIgURE 8: The deep sky objects inside and arround the error box of the OT GRB910219.

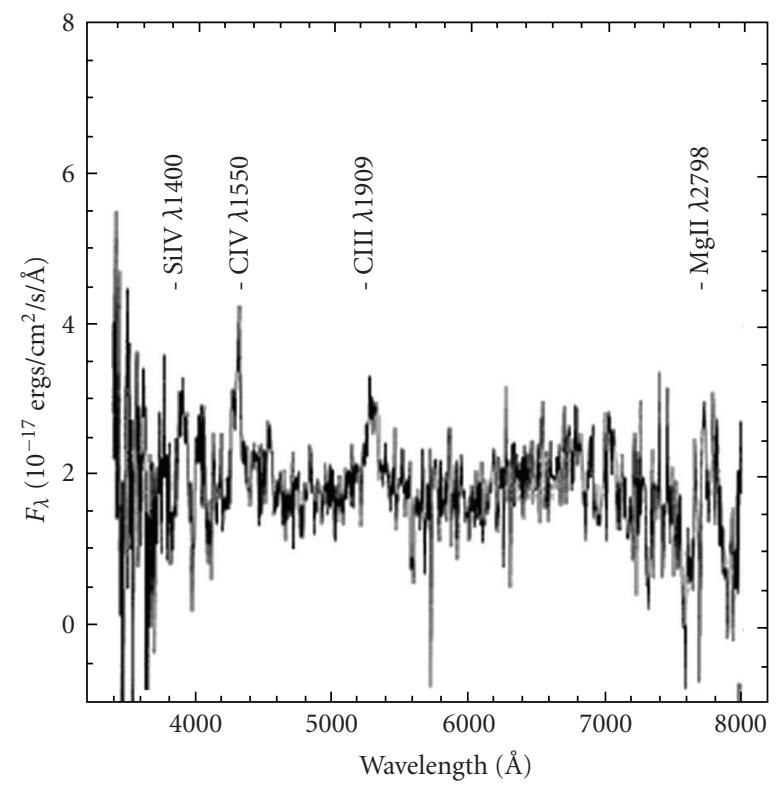

Figure 9: The spectrum of the object 41 inside the OT error box from previous figure. This is a candidate object for the OT, probably a faint QSO.

very probably faint AGN [8]. Possible explanation: large amplitude flares from faint AGN.

11.5. OT GRB910219 (Figures 7-9). This OT was found on the Harvard College Observatory (HCO) archival plate in the error box of GRB910219 [5, 6]. The image probably represents a large amplitude flare of a faint QSO at the OT position. Altogether 3.2 years of continuous optical monitoring (by archival plates) were used to reveal this object.

There still are many open questions related to the studies of GRB positions on astronomical plates. Before OTs of GRBs 


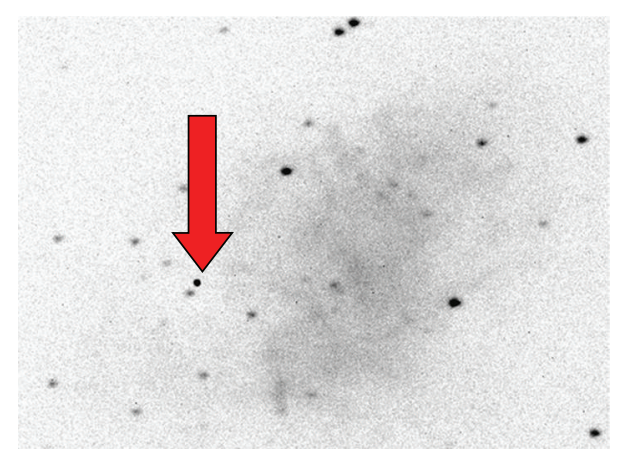

Figure 10: The OT of unknown nature: object detected on a sequence of CCD images (field M1) by the $60 \mathrm{~cm}$ telescope of the Brno Observatory by Filip Hroch. The estimated duration is less than $20 \mathrm{sec}$ and the magnitude $R=11$.

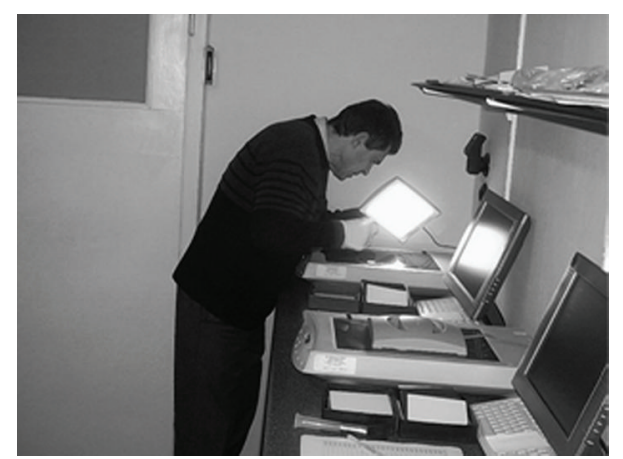

Figure 11: Plate scanning efforts at the Sonneberg Observatory, the largest European plate collection (280000 plates). More than 200000 plates were digitized already.

found by BeppoSAX, there were extended dedicated efforts to detect the optical emission of GRBs by taking Schmidt telescope plates hours to dozens of hours after GRBs triggers. No OTs or OAs were found at that time in relevant GRB error boxes with limiting magnitude between 16 and 22. Why; In recent understanding, there should be a positive detection at least in few cases.

11.6. OTs with CCD Detections. OTs with unknown origin were found not only on photographic plates but also on CCD images as shown in Figure 10.

\section{Future Prospects}

Over the last years, extended efforts were done at several major plate centres, such as Sonneberg, HCO, or PARI (see Figure 11). What is new in data mining in astronomical plate archives: (1) wide digitization of the plates and easy access to data recorded in astronomical emulsions, (2) specially designed and commercially available flatbed scanners, (3) dedicated programs to search in plate logs and catalogues, and (4) dedicated computer programs to evaluate data.

In the near future, we plan some additional improvements in the procedures and analyses described above, based on novel methods of image analysis (not only for direct images but also for spectral ones as well as multiple images), in particular,

(1) the evaluation of source image data from real image detector, that is, the data deconvolution for the compensation of distortions caused by the properties of the image sensor and optical and archiving (e.g., [9]),

(2) postprocessing of input image data (improving the signal/noise ratio, etc.),

(3) extraction of required data (data mining) from the images (e.g., analysis of spectral lines etc., e.g., [10]),

(4) statistical evaluation of obtained data,

(5) compression of image data for transfer and archiving (e.g., [11]),

(6) simulations of some parts of imaging/detecting chain (e.g., [12]),

(7) the program selection and recognition of important image information and comparison of their quantitative characteristics and reliability of the analysis based on analyses of series of multispectral images,

(8) statistical evaluation of large sets of multispectral image data sets, including those focused on particular triggers,

(9) the correlation of characteristics, selection of similar or belonging to the same type objects, automated searches for analogous objects on series of images. Possibility of stereoscopic analysis taking the astrometric results into account,

(10) improvements also includes development of algorithms and codes for automated analyses of low dispersion spectra, automated classifications, analyses of spectra lines, spectral continuum distributions, searches for spectral variability (both lines and continuum), and searches for objects with spectral variability and with peculiar spectra, and so forth $[10,13]$.

\section{Conclusions}

An alternative cost-effective method for classification and optical identification of INTEGRAL sources (and gammaray and hard X-ray sources in general) was discussed with some examples. This method can be applied both to detailed analyses of already identified sources as well as to identification of nonclassified new sources. Dedicated software programs are in design and development. The astronomical archival plates represent, due to large FOVs and large time coverage, an important data source for the monitoring of transient universe in general. For a given sky region, a total cumulative time of more than 3 years can be easily analysed on plates from several plate archives, impossible by any other method. Due to large FOV of astronomical archival plates, such large time coverage can be obtained for many of the celestial positions. The novel methods of image analyses and 
dedicated software packages are expected to further improve the importance of these data.

\section{Acknowledgments}

The authors acknowledge the support provided by ESA PECS 90823 and the Grant Agency of the Czech Republic, grants 205/08/1207 and 102/09/0997. The plate analyses were supported also by MSMT project ME09027. The investigation of Bamberg sky patrol plates is supported by DAAD-25-CZ4/08 project DAAD-AVCR.

\section{References}

[1] A. J. Bird, A. Malizia, A. Bazzano, et al., "The third IBIS/ISGRI soft gamma-ray survey catalog," Astrophysical Journal, Supplement Series, vol. 170, no. 1, pp. 175-186, 2007.

[2] N. Massetti, et al., "Unveiling the nature of INTEGRAL objects through optical spectroscopy. V. Identification and properties of 21 southern hard X-ray sources," Astronomy \& Astrophysics, vol. 459, no. 1, pp. 21-30, 2006.

[3] R. Hudec, et al., "Possible optical transient in Triangulum and its relation to the gamma-ray burst sources," Astronomy \& Astrophysics, vol. 225, p. 411, 1989.

[4] B. E. Schaefer, "Probable optical counterpart of a $\gamma$-ray burster," Nature, vol. 294, no. 5843, pp. 722-724, 1981.

[5] R. Hudec, et al., "OT 1928: A real candidate for GRB 781119," Astronomy \& Astrophysics, vol. 284, p. 499, 1994.

[6] R. Hudec, et al., "Optical studies in the fields of gamma ray burst sources. 2: GRB 910219 and the detection of flashing optical counterpart," Astronomy \& Astrophysics, vol. 284, p. 839, 1994.

[7] R. Hudec, et al., "Grain depth distribution and the reality of optical transient candidates near the GRB 790325b position," Astronomy \& Astrophysics, vol. 270, p. 151, 1993.

[8] C. Motch, R. Hudec, and C. Christian, "Quiescent candidates for the repeating optical transient OT $1809+31$," Astronomy \& Astrophysics, vol. 235, no. 1-2, pp. 185-196, 1990.

[9] M. Klíma, J. Pazderák, and K. Fliegel, "Examples of subjective image quality enhancement in multimedia," in Applications of Digital Image Processing XXX, A. G. Tescher, Ed., vol. 6696 of Proceedings of SPIE, pp. 1-9, San Diego, Calif, USA, August 2007.

[10] L. Hudec, Algorithms for spectral classification of stars, BSc thesis, Charles University Prague, Prague, Czech Republic, 2007.

[11] P. Pata, et al., "PCA Based Compression Technique for the BOOTES Image Data," in Proceedings of the 5th INTEGRAL Workshop on the INTEGRAL Universe (ESA SP-552 '04), V. Schönfelder, G. Lichti, and C. Winkler, Eds., p. 883, Munich, Germany, February 2004.

[12] M. Řeřábek, P. Páta, K. Fliegel, J. Švihlik, and P. Koten, "Space variant point spread function modeling for astronomical image data processing," in Astronomical Adaptive Optics Systems and Applications III, R. K. Tyson and M. Lloyd-Hart, Eds., vol. 6691 of Proceedings of SPIE, pp. 1-10, San Diego, Calif, USA, August 2007.

[13] R. Hudec, V. Šimon, and L. Hudec, "The GRB Investigations by ESA Satellite Gaia, in GAMMA-RAY BURST: Sixth Huntsville Symposium," in AIP Conference Proceedings, vol. 1133, pp. 52-54, 2009. 

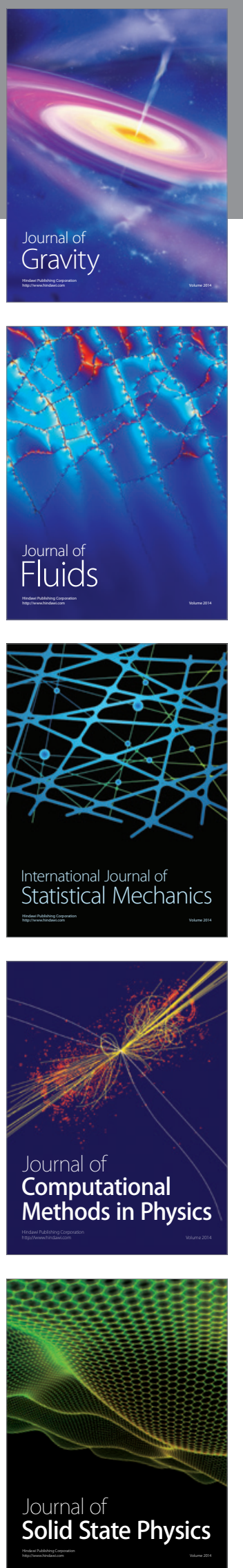

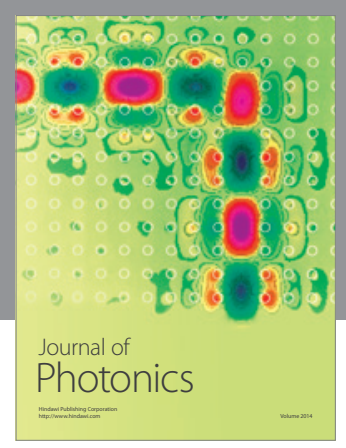

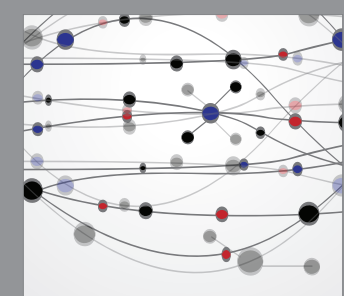

The Scientific World Journal
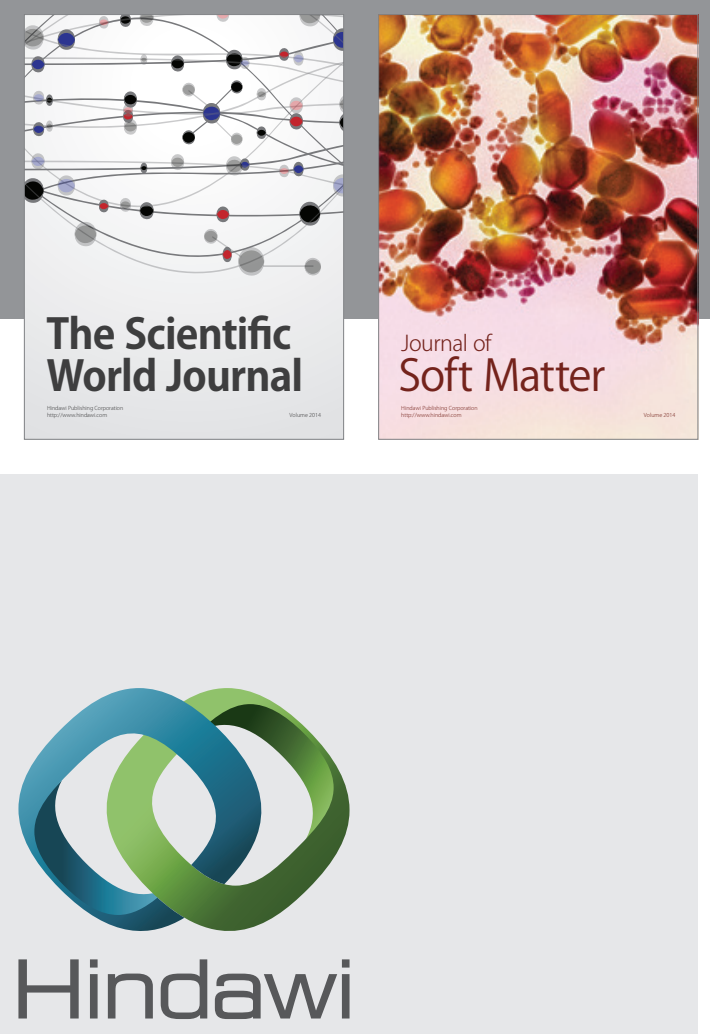

Submit your manuscripts at

http://www.hindawi.com
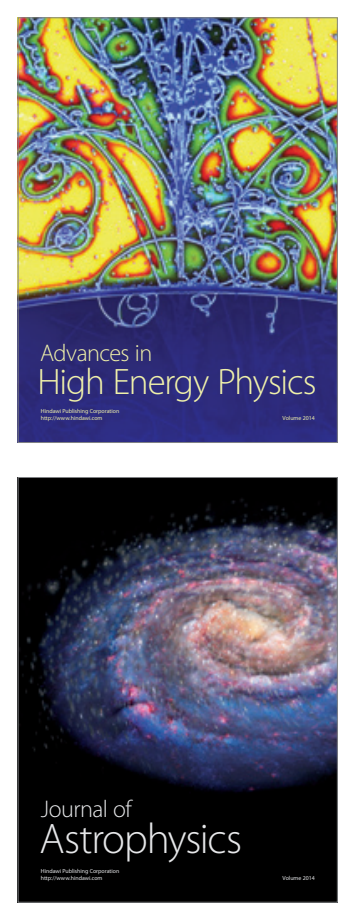
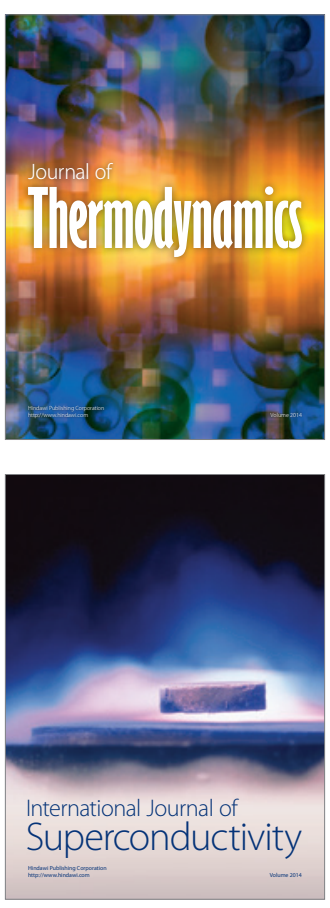
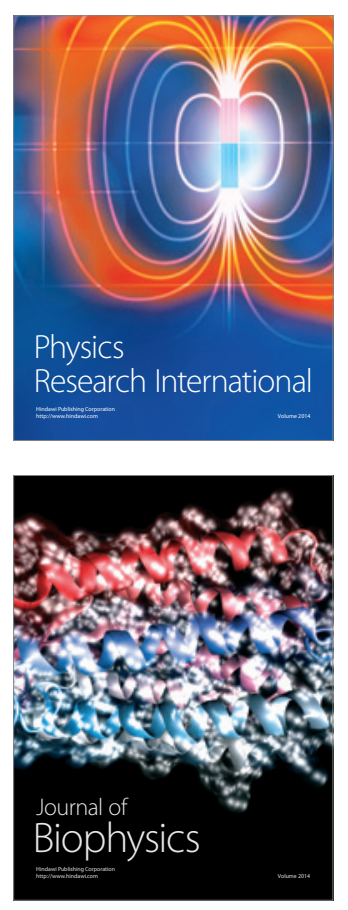
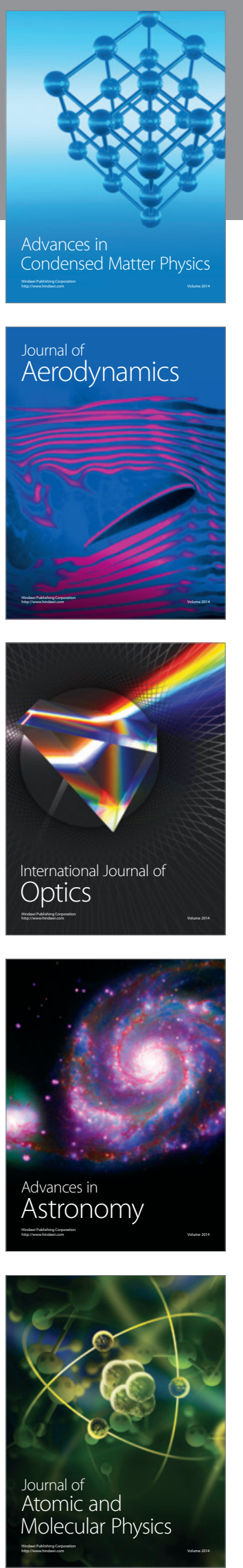